OPEN ACCESS

Edited by:

Alister Craig,

Liverpool School of Tropical Medicine,

United Kingdom

Reviewed by:

Catherine A. Gordon,

The University of Queensland,

Australia

Paulo J. G. Bettencourt, Catholic University of Portugal,

Portugal

*Correspondence:

Himanshu Gupta himanshu.gupta@/shtm.ac.uk

Samuel C. Wassmer

sam.wassmer@/shtm.ac.uk

Specialty section:

This article was submitted to

Parasite and Host,

a section of the journal

Frontiers in Cellular and Infection

Microbiology

Received: 12 October 2021 Accepted: 09 November 2021 Published: 16 December 2021

Citation:

Gupta H and Wassmer SC (2021) Harnessing the Potential of miRNAs in

Malaria Diagnostic and Prevention.

Front. Cell. Infect. Microbiol. 11:793954.

do: 10.3389/fcimb.2021.793954

\section{Harnessing the Potential of miRNAs in Malaria Diagnostic and Prevention}

\author{
Himanshu Gupta ${ }^{*}$ and Samuel C. Wassmer* \\ Department of Infection Biology, London School of Hygiene and Tropical Medicine, London, United Kingdom
}

Despite encouraging progress over the past decade, malaria remains a major global health challenge. Its severe form accounts for the majority of malaria-related deaths, and early diagnosis is key for a positive outcome. However, this is hindered by the non-specific symptoms caused by malaria, which often overlap with those of other viral, bacterial and parasitic infections. In addition, current tools are unable to detect the nature and degree of vital organ dysfunction associated with severe malaria, as complications develop silently until the effective treatment window is closed. It is therefore crucial to identify cheap and reliable early biomarkers of this wide-spectrum disease. microRNAs (miRNAs), a class of small non-coding RNAs, are rapidly released into the blood circulation upon physiological changes, including infection and organ damage. The present review details our current knowledge of miRNAs as biomarkers of specific organ dysfunction in patients with malaria, and both promising candidates identified by pre-clinical models and important knowledge gaps are highlighted for future evaluation in humans. miRNAs associated with infected vectors are also described, with a view to expandind this rapidly growing field of research to malaria transmission and surveillance.

Keywords: miRNAs, malaria, Plasmodium, Anopheles, biomarkers, diagnosis

\section{HIGHLIGHTS}

- miRNAs have recently emerged as essential regulators of immunity against Plasmodium parasites both in mosquito vectors and human hosts

- Specific miRNAs have been associated with severe malaria and its associated life-threatening complications

- miRNAs present promising diagnostic/prognostic biomarkers of malaria disease and may also be targeted by therapeutic interventions

\section{INTRODUCTION}

Despite encouraging control progress over the past decade, malaria remains a major global health challenge. 409,000 malaria-associated deaths were reported in 2019, and 94\% of these occurred in Africa (WHO, 2020). In addition to endemic countries, fatal cases are also reported in other parts of the world due to increasing imported infections (Zoller et al., 2009; Mischlinger et al., 2020). Malaria can be caused by several Plasmodium species in humans, including Plasmodium falciparum (Pf), P. vivax (Pv), P. knowlesi (Pk), P. ovale (Po), and P. malariae (Pm) (Garcia, 2010). Among these, 
$P f$ and $P v$ are responsible for majority of cases worldwide (WHO, 2020), and $P f$ is considered the most lethal of the human malaria parasites. $P f$ cases can be classified into severe and uncomplicated malaria based on the World Health Organization (WHO) criteria (WHO, 2014). Severe falciparum malaria is characterized by multi-organ dysfunctions, which are triggered by the sequestration of infected erythrocytes (iEs) within the microvasculature of the host, combined with an exaggerated production of inflammatory mediators (White et al., 2013; Milner et al., 2014). In contrast, $P v$ infection has been perceived as relatively benign until recently, despite documented debilitating and potentially life-threatening complications (Gupta et al., 2015a; Gupta et al., 2016a; Anvikar et al., 2020). Severe malaria (SM) cases have also been reported in patients infected with $P k$ (Cox-Singh et al., 2010), Po (Kotepui et al., 2020a), and Pm (Kotepui et al., 2020b), although these remain anecdotal. There are distinct biochemical and morphological features across the different Plasmodium species, but the mechanisms underlying the development of SM are likely to be similar. They all involve inflammation caused by the release of Plasmodium-derived components (known as pathogen-associated molecular patterns), as well as host-derived components (or damage-associated molecular patterns) and sequestration of iEs in some specific species (Gazzinelli et al., 2014). Plasmodium spp. are transmitted by female Anopheles mosquitoes, which inject sporozoites into the subcutaneous tissue of the human host during their blood meal, triggering the host phase of the cycle (White, 2017).

The fundamental pathogenesis of SM is still poorly understood and treatments are currently precariously limited to antimalarial drugs and emergency supportive care (White et al., 2014). An early diagnosis is key for prompt and accurate treatment, resulting in positive outcomes. Unfortunately, this scenario remains rare as SM still have a fatality rate of $15-30 \%$ when patients are appropriately treated upon admission (Lucchi et al., 2011). The reasons are manifold and include i) late presentation to the hospital (the damage is already done); ii) generic symptoms such as a fever that overlaps with the presentation of viral, bacterial and parasitic infections (the infection is missed) (Rubio et al., 2016); and iii) out-of-criteria patients with laboratory parameters just below or above the hard WHO cut-offs for disease severity at the time of admission (the disease severity is increasing but unnoticed). For the latter category, patients treated for uncomplicated malaria (UM) have been reported to be ill enough to warrant hospitalization (Sahu et al., 2020), and could then progress to develop lifethreatening SM. This suggests that complications may develop silently until it is too late (the damage is already done). A parallel study showed that UM patients with delayed treatment are highly likely to develop SM (Mousa et al., 2020). In view of these challenges, it is crucial to identify new biomarkers of early SM that can be then developed into cheap, reliable diagnostic and/or prognostic tools for clinicians to identify patients at risk.

microRNAs (miRNAs), a class of small non-coding RNAs (18-24 nt length), are rapidly released into the blood circulation upon physiological changes, including infection and organ damage (Cortez et al., 2011). They regulate gene expression endogenously at the post-transcriptional level, either through translation repression or mRNA degradation (Cortez et al., 2011). miRNAs can be secreted extracellularly as bound to lipoproteins or within cell-derived extracellular vesicles (Valadi et al., 2007; Arroyo et al., 2011; Nik Mohamed Kamal NNSB and Shahidan WNS 2020). These small molecules are highly stable and can be detected in a wide range of biological fluids, making them exceptionally promising non-invasive biomarkers (Rubio et al., 2016). Indeed, they can be potentially used to not only detect an infection, but also diagnose early-stage tissue or organ damage. This would represent a significant advantage over the standard methods currently being used to diagnose malaria, such as microscopic examination of blood smears (Fleischer, 2004), plasma antigen detection (Spencer et al., 1979), rapid diagnostic test (Moody and Chiodini, 2002) and molecular conventional and quantitative PCR assays (Snounou et al., 1993; Gupta et al., 2016b).

For these reasons, researchers using in vitro and mice models, as well as clinical samples have attempted to identify miRNAbased biomarkers of malarial disease in the last decade, with the hope to develop new diagnostic and prognostic tools. Remarkably, several miRNAs have also been discovered in Pfinfected Anopheles mosquitoes. Thus, specific profiles of miRNAs associated with different phases of the malaria life cycle have the potential to help control and elimination efforts by allowing the identification of (i) carrier mosquitoes, (ii) infected patients, symptomatic or not, and (iii) potential tailored treatment for the former category. The present review covers our current knowledge of miRNAs described across the malaria parasite life cycle, including both vector and host. Their potential as biomarkers of acute and chronic pathologies associated with malaria disease is discussed, as well as future research recommendations for this promising and rapidly growing field of research.

\section{BUZZING AROUND: THE VECTOR PHASE}

To complete the malaria parasite life cycle, gametocytes are ingested by female Anopheles mosquitoes from the blood of infected human hosts. Parasites then invade mosquito midgut, thereby eliciting a physiological response leading to variations in the expression and release of various miRNA. Studies of infected anopheline mosquitoes have identified several miRNAs (Figure 1a) that may alter mosquito immunity against Plasmodium infection. These miRNAs also have important roles in the development and maturation of the parasite within their vectors, and distinct expression patterns of miRNAs in specific tissues have been described (Winter et al., 2007; Mead and Tu, 2008; Jain et al., 2014; Dennison et al., 2015; Lampe et al., 2019; Dong et al., 2020). One study reported a reduction in the expression of aga-miR-34, aga-miR-1174 and aga-miR-1175 miRNAs in the midgut of $P$. berghei-infected Anopheles gambiae compared controls. In contrast, aga-miR-989 levels were significantly increased in the presence of the murine 
malaria parasite $P$. berghei. aga-miR-989 levels decreased significantly in the rest of the body, suggesting the specificity of aga-miR-989 to the midgut of infected Anopheles gambiae. Additionally, knocking down Dicer1 and Ago1 mRNAs enhanced the vector's sensitivity to Plasmodium infection (Winter et al., 2007). Another study identified a series of differentially expressed Anopheles gambiae miRNAs induced by normal blood meal (miR-7, miR-92a, miR-317, and miR-N3) and infectious blood meal (miR-N3, miR-317, miR-2940, miRN5, miR-N6 and miR-N4) (Biryukova et al., 2014). Similarly, elevated levels of aga-miR-989 and aga-miR-305 miRNAs were found in $P f$-infected mosquito midgut tissue compared to naïve ones (Figure 1a). Furthermore, aga-miR-305 inhibition increased resistance to Pf infection (Dennison et al., 2015). Remarkably, bloodmeals have been shown to induce miR-276$5 \mathrm{p}$ levels in mosquito, which regulate the expression of branched-chain amino acid transferase to terminate the reproductive cycle. Inhibition of miR-276 elongated high rates of amino acid (AA) catabolism and increased female mosquito fertility, suggesting that timely termination of AA catabolism restricts both mosquito investment into reproduction and development of the transmissible sporozoite forms (Lampe et al., 2019). miRNAs (aga-miR-8, aga-miR-14, and aga-miR305) were found to regulate mosquito immunity against parasite infection. Depletion of aga-miR-14 or aga-miR-305, but not agamiR-8, increased mosquito resistance to both $P$. berghei and $P f$ infection by enhancing the expression of multiple immunityrelated and anti-Plasmodium genes. This indicates a potential role for mosquito miRNAs in the development of malaria control through genetically engineered vectors (Dong et al., 2020). miRNA profiling revealed distinct expression patterns of miRNAs from early embryo to adult stages in Anopheles stephensi. miR-x2 was found associated with female reproduction, and constant miR-14 expression was indicative of its importance across all mosquito life stages (Mead and Tu, 2008). By applying next-generation sequencing (NGS) technology to whole vectors, differentially expressed miRNAs were reported post blood feeding (13 miRNAs) and parasite infection (16 miRNAs) in Anopheles stephensi. A set of miRNAs showed significant expression changes between $42 \mathrm{~h}$ (midgut invasion) and 5 days (sporozoites release) post-infection, highlighting a stage-specific parasite influence on vector miRNAs. These miRNAs are known to target genes involved in several metabolic pathways including metabolic, redox homeostasis and protein processing machinery components. Specific miRNAs (miR-124, 305, and 309; Figure 1a) regulate multiple immune pathway genes (Jain et al., 2014). aan-miR-92a and aan-miR-275 levels were found upregulated and downregulated in blood-feeding and Plasmodium infection (Figure 1a), respectively, in the midgut of Anopheles anthropophagus compared to sugar-feeding mosquitoes (Liu et al., 2017). Collectively, these findings suggest that the expression of mosquito miRNAs changes in response to Plasmodium infection, and could therefore be used in molecular assays such as RT-qPCR to screen and identify parasite-carrying vectors. This would be done by comparing

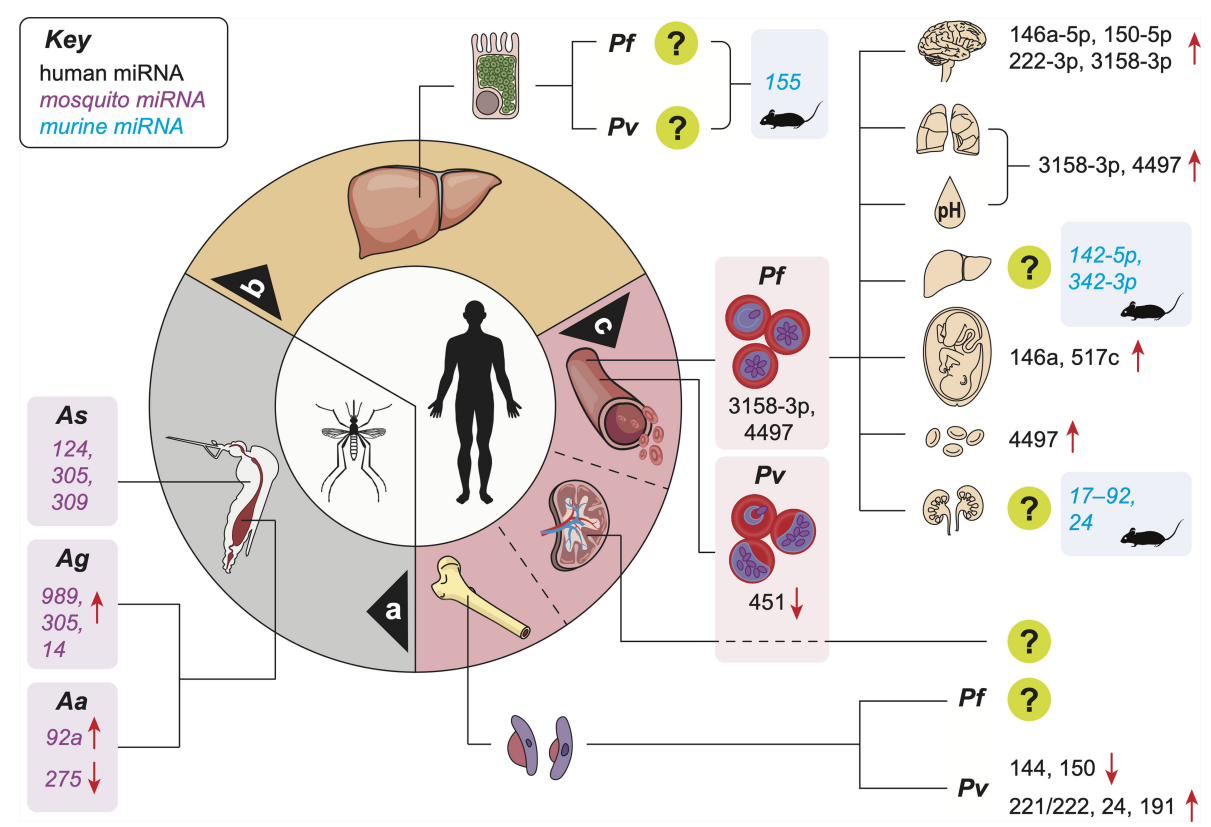

FIGURE 1 | Reported miRNA expression changes in the vector and host during malaria infection. miRNA level changes in the midgut of infected mosquitoes a; in animal models of the infection during the silent hepatic phase $\mathbf{b}$; and in patients during the symptomatic erythrocytic phase $\mathbf{c}$. Organ and metabolic dysfunctions represented in the upper right quadrant are, from top to bottom: brain (cerebral malaria), lungs (ARDS), acidosis (hyperlactatemia), liver (jaundice), placenta (PAM), decreased red blood cell count (anemia), kidneys (AKI). [upregulation ( $\uparrow$ ); downregulation ( $\downarrow$ ); ?: human miRNAs not identified; Pf, Plasmodium falciparum; PV, Plasmodium vivax; Aa, Anopheles anthropophagus; Ag, Anopheles gambiae; As, Anopheles stephensi]. 
their miRNA levels against a baseline from uninfected vectors of the same species. In turn, such approach could help estimating metrics of exposure and transmission intensity (Tusting et al., 2014). Epidemiological and ecological studies of malaria traditionally utilize detection of Plasmodium sporozoites in whole mosquitoes or salivary glands by microscopy (Habluetzel et al., 1992), or serological (Wirtz et al., 1987) or molecular assays (Echeverry et al., 2017; Calzetta et al., 2018). However, these methods are time-consuming, require skill and expertise, are labour-intensive, and can over- or underestimate mosquito transmission potential (Ramirez et al., 2019). Thus, there is a need for new technologies to improve mosquito surveillance programmes, and miRNA-based assays could accurately detect infected mosquitoes in a short period of time, with the potential to inform decision-making in the fight against malaria.

\section{HIDE: THE ASYMPTOMATIC PRE- ERYTHROCYTIC PHASE}

Upon the bite of an infected vector, sporozoites located in its salivary glands are injected into the subcutaneous vasculature of the host. Once in the bloodstream, they travel to the liver and infect hepatocytes, initiating the silent phase of the infection, during which each sporozoite develops into hundreds of merozoites. This pre-erythrocytic phase cannot currently be identified in infected patients, but represent a pivotal window of opportunity for diagnostic and treatment, since transmission does not start until the erythrocytic stage is initiated. Identifying infected individuals at this stage could revolutionize the active surveillance programme and represent a powerful tool in malaria control. A study of the murine model of malaria infection using $P$. berghei parasites identified several miRNA candidates associated with this pre-erythrocytic phase. Indeed, murine infection with genetically attenuated parasites (GAPs) that arrest in the liver and induce sterile immunity leads to the upregulation of miR-155 levels in the liver (Figure 1b), particularly in non-parenchymal cells including liver-resident macrophages, or Kupffer cells (Hentzschel et al., 2014). Additional studies are warranted to investigate plasma miRNA profiles specifically associated with this phase, which could be used in RT-qPCR-based assays for the early identification of silent infections.

\section{AND SEEK: THE SYMPTOMATIC ERYTHROCYTIC PHASE}

After release from infected hepatocytes, merozoites invade erythrocytes and mature from ring stages to trophozoites, and ultimately, to schizonts (Figure 1c). The burst of iEs to release daughter merozoites results in exponential growth of the parasite population, and some of these merozoites develop into sexual forms, or gametocytes. The clinical symptoms of malaria are mainly attributed to the rupture of schizont stage-iEs and the subsequent release of parasite-derived toxins, which stimulate innate immune cells to produce cytokines and inflammatory mediators causing periodic episodes of febrile illness (Oakley et al., 2011; Gazzinelli et al., 2014). Circulating cytokines also induce the upregulation of adhesion molecules by endothelial cells which, in turn, increase parasite sequestration (Gazzinelli et al., 2014). This phenomenon is mediated by Pf erythrocyte membrane protein 1 (PfEMP1), a family of proteins present on the surface of iEs and encoded by approximately 60 var genes (Hviid and Jensen, 2015). PfEMP-1 can bind to several host receptors on the surface of capillary endothelium, uninfected erythrocytes, placental syncytiotrophoblasts, and platelets (Rowe et al., 2009; Turner et al., 2013; Wassmer et al., 2015). Such receptors include intercellular adhesion molecule-1 (ICAM-1), CD36, endothelial protein $\mathrm{C}$ receptor (EPCR), gC1qR, chondroitin sulfate A (CSA), or complement receptor 1 (Jensen et al., 2020). By binding to the vessel walls, mature forms of $\mathrm{Pf}$ remove themselves from the circulation, thereby avoiding clearance by the spleen (Cranston et al., 1984; Buffet et al., 2011). However, the local accumulation of iEs disrupts or completely abrogates the blood flow, promotes clotting, triggers endothelial cell and blood-brain barrier disruption, potentially leading to the extravasation of vascular content in the parenchymal tissue and increased local inflammation. These mechanisms have all been linked to SM (Gazzinelli et al., 2014).

Cell-cell interactions, as well as the immune response of the host are highly likely to influence the expression of miRNAs during malaria infection. Remarkably, only human miRNAs are found in Pf-iEs (Rathjen et al., 2006; Xue et al., 2008), and it has been reported that human erythrocytes miRNAs could translocate and integrate into the parasite messenger RNAs to block their translation (LaMonte et al., 2012). In particular, miR-451 and let-7i were found abundant in sickle erythrocytes, and together with miR223, reduced parasite growth (LaMonte et al., 2012).

A study using controlled human $P f$-infection in adults identified a profile of 84 miRNAs associated with $\mathrm{T}$ cell and $\mathrm{B}$ cell activation, indicating a pivotal role of miRNAs in interindividual variability in the immune response to falciparum malaria. Volunteers with increased levels of miR-15a-5p, miR$30 c-5 p$, and miR-30e-5p had a higher frequency of activated and proliferating $\mathrm{T}$ cells and could control their Pf parasitemia more effectively after infection (Burel et al., 2017).

Similarly, host genetics can regulate $P f$ infection (Gupta et al., 2013; Gupta et al., 2015b; Gupta et al., 2017a), and the protective effect of rs114136945 minor allele on parasitemia mediated through miR-598-3p expression was recently reported (Dieng et al., 2020). In a study carried out in Thailand, the downregulation of both miR451 and miR-16 was reported in 19 malaria patients compared to healthy individuals (Chamnanchanunt et al., 2015). Subsequent work on a smaller number of participants found 8 differentially expressed miRNAs between Pf malaria patients and healthy controls (Bertrams et al., 2021).

\section{Severe Malaria: Multi-Organ and Physiological Dysfunctions}

Severe $P f$ malaria is characterised by the sequestration of iEs in the microvasculature of the host (Miller et al., 2002). Combined 
with an exacerbated production of inflammatory mediators, this accumulation leads to the dysfunction of peripheral organs, either alone or in combination, leading to severe complications. These include acute respiratory distress syndrome, (ARDS, affecting the lungs), jaundice (liver), acute kidney injury (AKI, kidneys) or cerebral malaria (CM, brain) (White et al., 2013; Milner et al., 2014). The hard WHO cut-offs for these severe complications at the time of admission exclude patients with parameters just above or below the defined threshold. Thus, determining direct or indirect markers of early and still progressing organ dysfunction in the context of SM could revolutionize its clinical management. A recent, large-scale study using a combination of in vitro models and plasma samples from Mozambican children infected with Pf and diagnosed with SM or UM demonstrated for the first time the association between both hsa-miR-3158-3p and hsa-miR4497 with SM, its complications, and $P f$ biomass (Figure 1c) (Gupta et al., 2021a). These findings suggest that although unable to produce miRNAs (Xue et al., 2008), Plasmodium may manipulate the production of host miRNAs. In turn, these changes in small molecule levels have the potential to not only shed light on the molecular mechanisms involved in the pathogenesis of SM, but also be used as signatures associated with individual complications. As SM is a broad spectrum disease, such miRNA signatures may help identify complications and tailor treatment approaches to increase survival.

\section{Neurological Changes: Cerebral Malaria (CM)}

$\mathrm{CM}$ is an acute neurological complication and often lethal form of SM. It has a fatality rate up to $30 \%$ in treated patients (Wassmer et al., 2015), and neurocognitive sequelae are frequent in survivors (WHO, 2014). CM cases are predominantly seen in African children under five, as the high malaria transmission intensity in sub-Saharan Africa leads to the development antimalarial immunity during childhood. However, in South East Asia, where malaria transmission is seasonal and not intense enough to induce robust immunity, CM cases are mainly found in older children and adults (Sahu et al., 2015). In addition, while CM is mainly accompanied by severe anemia and/or metabolic acidosis in African children, it is very often reported in combination with other organ involvement such as lungs, liver and kidneys leading to ARDS, jaundice and AKI, respectively in Asian adults (Wassmer et al., 2015). The mechanisms behind these distinct clinical features between African children and South East Asian adults is still poorly understood.

In addition, CM is challenging to diagnose using the tools currently available. An autopsy study in Malawi demonstrated that up to $23 \%$ of children clinically diagnosed with CM had, in fact, another cause of death based on post-mortem examination (Taylor et al., 2004). Further work reported that CM was indeed overly misdiagnosed when assessed by parasite load alone; 38\% versus only $1 \%$ who truly fulfilled WHO criteria (Makani et al., 2003). Over the past decade, neuroimaging studies in endemic areas have improved our understanding of CM (Mohanty et al., 2014). Distinct age-dependent brain changes identified by magnetic resonance imaging (MRI) on admission were recently linked to poor outcomes. In pediatric CM, severe brain swelling with brain stem herniation was associated with fatality (Seydel et al., 2015), a feature not observed in fatal adult cases (Mohanty et al., 2011; Maude et al., 2014). Our team demonstrated that in the latter group, global cerebral hypoxic injury was associated with mortality (Sahu et al., 2020). While the early identification of such features may prove critical to inform clinical management and improve survival in patients at risk, access to MRI facilities remains extremely limited in malaria endemic countries due to operational, logistical and financial challenges (Latourette et al., 2011). In addition, the occurrence of specific pathogenic features in different age groups may be difficult to detect and differentiate in adolescents or young adults. Therefore, alternative biomarkers of brain changes identified by MRI and validated in both age groups would represent a powerful prognosis tool to identify patients at risk of developing fatal disease, inform clinical management, and decrease mortality in CM. In view of these challenges, miRNAs found associated with defined MRI features could be helpful in CM diagnosis.

miR-155 was found to be an important player in the CM pathogenesis via negative regulation of blood-brain-barrier integrity and $\mathrm{T}$ cell function (Barker et al., 2017). In addition to miR-155, murine studies have yielded a series of biomarker candidates for CM, including miR-19a-3p, miR-540-5p, miR223-3p, miR-142-3p, miR-19b-3p, let-7i, miR-27a, miR-150, miR-146a, miR-193b, miR-205, miR-215 and miR-467a (ElAssaad et al., 2011; Cohen et al., 2018; Martin-Alonso et al., 2018). These miRNAs are significantly involved in several pathways relevant to $\mathrm{CM}$, including TGF- $\beta$, inflammation, TNF signaling, monocyte sequestration in cerebral microvessels, endocytosis (El-Assaad et al., 2011; Cohen et al., 2018; MartinAlonso et al., 2018). Among the miRNA candidates identified, miR-146a-5p and miR-150-5p were also associated with CM in Indian patients (Gupta et al., 2021b), together with miR-222-3p and miR-3158-3p (Figure 1c). Upon further analysis, high miR150-5p and miR-3158-3p levels were associated with fatal CM. miR-3158-3p levels decreased significantly in CM survivors at day 30 post-treatment, strongly suggesting the CM specificity of this miRNA. Lastly, miR-3158-3p levels were found correlated with hypoxia in the brain of adults, and negatively correlated with increased brain volume of children, both identified by MRI (Gupta et al., 2021b). This indicates that the production of miR$3158-3 p$ is decreased in CM patients with high brain volume on admission, a feature associated with a poor outcome in children (Seydel et al., 2015; Sahu et al., 2020). Inversely, miR-3158-3p levels increased in patients with high hypoxia on admission, a hallmark of fatal adult CM (Sahu et al., 2020). While further validation of the association between miR-3158-3p levels and MRI features of poor outcomes in CM is needed, our findings support the potential use of miR-3158-3p for CM prognosis in children and adults in lieu of neuroimaging (Gupta et al., 2021b).

\section{Liver Dysfunction}

Several studies using the murine model of $P$. chabaudi infection identified differentially expressed miRNAs in the liver of infected 
animals (Delic et al., 2011; Al-Quraishy et al., 2012; Dkhil et al., 2016). An upregulation of three and a downregulation of 16 miRNAs was reported in the mouse liver following infection. Remarkably, miRNA expression pattern persisted in immune mice even after re-infection, suggesting that the development of a protective immunity against $P$. chabaudi infection may be regulated in part by miRNAs (Delic et al., 2011). Another study reported 169 miRNAs downregulated in liver tissue obtained from $P$. chabaudi-infected mice, while miR-142-5p and miR-342-3p were found upregulated (Figure 1c) (AlQuraishy et al., 2012). Similarly, a downregulation of 18 and upregulation of 14 miRNAs were reported in the liver during the acute phase of $P$. chabaudi infection (Dkhil et al., 2016).

\section{Placenta: Pregnancy-Associated Malaria (PAM)}

Pregnancy-associated malaria (PAM) is defined by the sequestration of iEs in the placental intervillous spaces. This sequestration is mediated by binding of VAR2CSA (Salanti et al., 2003) to chondroitin sulfate A (CSA) expressed at the surface of the placental syncytiotrophoblast (Zakama et al.; Fried and Duffy, 1996), contributing to inflammatory infiltrates and reduced nutrient transfer to the fetus (Rogerson et al., 2018), ultimately causing adverse outcomes including low birth weight, preterm birth, stillbirth, and miscarriage (Nosten et al., 2004). In addition, women who are pregnant for the first time generally lack immunity to antigenic variant presented by $P f$ parasites that accumulate selectively in the placenta, which put them at a higher risk of infection compared to non-pregnant women (Brabin et al., 2004; Gamain et al., 2007). The risk of infection decreases in the second trimester compared to first trimester in primigravid women. The infection risk also decreases after successive pregnancies (Gamain et al., 2007). Similarly to other organ-specific dysfunctions induced by $P f$, increased levels of miR-517c (Figure 1c), an immunomodulator in pregnancy and tumorigenesis (Bullerdiek and Flor, 2012), were reported in mothers with PAM when compared to non-infected controls (Moro et al., 2016). miR-146a rs2910164 polymorphism was found to increases the odds of PAM occurrence in primigravid Ghanaian women, suggesting a role for miR-146a in this complication (van Loon et al., 2019). The authors of the study suggested that miR-146a is involved in protective malarial immunity, particularly its innate component.

\section{Other Organ and Physiological Dysfunctions}

A limited number of studies have been carried out to identify miRNAs associated with SM complications and organ dysfunction such as acidosis, severe anemia, ARDS, or AKI. Higher levels of miR-3158-3p were measured in Mozambican children who had acidosis or ARDS compared to UM children. In contrast, miR-4497 was associated with severe anemia and ARDS (Figure 1c) (Gupta et al., 2021a). A review suggested that miR-210; miR-125b and miR-181b; as well as miR 17-92 and miR-24 could be promising miRNA-based biomarkers of acidosis, ARDS and renal failure, respectively (Figure 1c) (Chamnanchanunt et al., 2017). However, miR-210-3p was not found associated with $\mathrm{SM}$ in a recent study conducted in India (Gupta et al., 2021b). Because this is still an emerging field, the data on biomarkers of malaria-associated organ dysfunction and severe complications remain limited. The resultant knowledge gap needs to be addressed to identify miRNAs associated with ARDS, AKI, acidosis and SA (Figure 1c). In turn, these tools will help researchers and clinicians to $i$ ) increase the granularity of the SM diagnosis and ii) determine pathogenetic pathways and potential therapeutic targets in SM (Wassmer et al., 2015). It is also noteworthy that grouping patients with single distinct SM complications is challenging. Indeed, acidosis, for example, is caused by the host anaerobic glycolysis due to tissue hypoxia following iE sequestration (Rubio et al., 2016) and develop in combination with other complications in most patients. Therefore, global or combination profiling may be an easier approach than identifying the profiles of miRNAs associated with specific dysfunction.

Thrombocytopenia is common in malaria (Lacerda et al., 2011; Anvikar et al., 2020) and has been associated with SM (Anvikar et al., 2020). The miRNA pair miR-4454/miR-7975 was found associated with low platelet counts (Santos et al., 2021). In addition, the roles of extracellular vesicles (EVs), small membrane-bound vesicles that can be classified based on their size, origin, and functions (EL Andaloussi et al., 2013), have recently emerged in the different stages of malaria life cycle, as well as in the pathogenesis of SM (Babatunde et al., 2020; Cheng et al., 2020). A high number of EVs can be found during pathological conditions (Combes et al., 2004; Nantakomol et al., 2011), and they can act as immunomodulators during Plasmodium infection either directly (Mantel and Marti, 2014) or via their miRNA cargo (Mantel et al., 2016; Wang et al., 2017). Indeed, EV contain miRNAs with regulatory functions, and those are protected from degradation by RNases (Cheng et al., 2014; Vojtech et al., 2014). A study reported that EV-derived miR-150-5p, miR-15b-5p and let-7a-5p were significantly up regulated in malaria patients compared to healthy individuals (Ketprasit et al., 2020). let-7a-5p miRNA was associated with falciparum malaria (Ketprasit et al., 2020), and miR-150-5p, miR-15b-5p and let-7a-5p with $P v$ infection (Ketprasit et al., 2020).

\section{Chronic Malaria}

In high transmission settings, intense exposure to malaria drives the development of anti-disease immunity, whereby individuals control their immune response to infection, ultimately resulting in asymptomatic malaria (AM) (Kimenyi et al., 2019). By definition, it is characterised by the lack of apparent clinical symptoms, and infected individuals do not seek treatment. They are missed by passive surveillance while remaining an important gametocytes reservoir, and such infections contribute to the persistence of malaria transmission (Galatas et al., 2016; Andolina et al., 2021). AM has been perceived as relatively benign until a study demonstrated that it was associated with recurrent episodes of symptomatic parasitemia, chronic anemia, maternal and neonatal mortality, co-infection with invasive bacterial disease, cognitive impairment, and continuous transmission (Chen et al., 2016). The authors proposed to rename the misleading AM to chronic malaria (Chen et al., 2016). 
As a large proportion of AM infections are associated with low-density parasitemia, these individuals are also likely to be missed by conventional tests such as microscopy and rapid diagnostic tests, which have limited detection sensitivity (Okell et al., 2012; Bousema et al., 2014). New approaches are therefore needed for large scale populations screening. miR-3158-3p and miR-4497 were found associated with parasite biomass (Gupta et al., 2021a) so it could be postulated that these miRNAs are promising candidate to identify sub-patent infections. However, further investigations are warranted to determine the parasitemia detection threshold afforded by these miRNAs.

In a similar way, $P v$ has also been assumed to be unharmful due to relatively low parasitemia levels and high proportions of $\mathrm{AM}$ cases. However, evidence is mounting that vivax malaria is debilitating and potentially causes life-threatening complications similar to the ones reported in Pf infection (Gupta et al., 2015a; Gupta et al., 2016a; Anvikar et al., 2020). miR-451 and miR-16 levels were reported downregulated in $P v$ patients compared to controls (Figure 1c) (Chamnanchanunt et al., 2015). The miRNA pair miR-4454/miR-7975 was upregulated while miR520f-3p, miR-150-5p and let-7b-5p were down regulated in patients with $P v$ infection (Santos et al., 2021). The miR-1505 p association with $P v$ infection has not been consistent in Thai and Brazilian populations (Ketprasit et al., 2020; Santos et al., 2021), suggesting the need for further validation. Another study found miR-7977, miR-28-3p, miR-378-5p, miR-194-5p and miR-3667-5p associated with $P v$ infection, and the authors postulated that miR-7977 exacerbated its pathology via the UBA52 or TGF-beta signalling pathways (Kaur et al., 2018). These results suggest that host miRNA levels are also influenced by the presence of $P v$ infection, which opens new avenues to identify miRNA-based biomarkers that can not only differentiate between malaria patients from healthy individuals but could also identify different Plasmodium species. To the best of our knowledge, this research field remains limited and only miR451 levels were found differentially expressed between patients infected with $P f(n=3)$ and $P v(n=16)$ (Chamnanchanunt et al., 2015). However, the sample size for this comparison was small. Combined Plasmodium species-specific miRNA-based biomarkers could play an important in the context of malaria elimination programs to detect multi-species infections. Currently, these programs are mainly focusing on $P f$, which can lead to the rise of other Plasmodium species.

Recent work demonstrated for the first time a large parasite biomass in the spleen of AM individuals compared to the peripheral circulation, which is likely to contribute to anemia (Kho et al., 2021a; Kho et al., 2021b). Both reticulocytes and asexual $P v$ parasites were observed in the splenic tissue, suggesting that the measure of the peripheral parasitemia alone vastly underestimates the $P v$ biomass in AM (Kho et al., 2021a). miR-4497 may play an important role in detecting hidden splenic parasites in AM individuals, as it was found associated with parasite biomass and SA (Gupta et al., 2021a). In addition, another study reported the downregulation of 25 miRNAs in the spleen obtained from mice infected with P. chabaudi (AlQuraishy et al., 2012). However, additional validation work is needed to identify miRNA-based biomarkers with a potential to detect splenic parasites in individuals with AM. This approach will be crucial to identify and treat such cases in order to interrupt malaria transmission.

A hallmark of $P v$ is its ability to form dormant liver stages, or hypnozoites. These can reactivate, causing recurrent episodes of malaria (Dayananda et al., 2018). However, frequent episodes do not allow patients to recover from hematological damage, leading to severe anemia (Tjitra et al., 2008). Because current diagnostic tools cannot detect hypnozoites, a 14-day course of primaquine is recommended by the $\mathrm{WHO}$ for all patients who are not deficient in the glucose-6-phosphate dehydrogenase (G6PD) enzyme (WHO, 2015). In view of the challenges associated with the wide implementation of a long treatment course and practical pretreatment testing to identify G6PDdeficient individuals at risk of severe hemolysis (Baird et al., 2016), a screening tool to detect these hypnozoites may be key to reduce these bottlenecks. miRNA-based assays could fill this gap and support current elimination efforts.

Overall, miRNAs found associated with the symptomatic phase of the malaria life cycle in different studies, specifically miR-146a-5p, miR-150-5p, miR-3158-3p and miR-4497 have the potential to develop diagnostic and prognostic tools to identify malaria patients, organ dysfunction, and patients at the risk of developing SM associated life-threating complications, inform their clinical management, and decrease SM associated mortality.

\section{READY FOR TAKE OFF: GAMETOCYTES}

Gametocytes are sexual forms of the malaria parasite, which enable the establishment of infection in mosquitoes from its mammalian host, a crucial step in malaria transmission (Bousema and Drakeley, 2011). Similarly to mature asexual parasites in iEs, it has been postulated that immature gametocytes are "sequestered" away from the peripheral blood and the host immune cells. This process would ensure their safe maturation before they are released into the circulation (Bousema and Drakeley, 2011). Indeed, a high prevalence and abundance of early $P f$ sexual stages was identified in the bone marrow (BM), which was linked to both dyserythropoiesis and severe anemia (Aguilar et al., 2014). It is noteworthy that the presence of gametocytes in the BM leads to transcriptional changes of miRNAs expression involved in erythropoiesis. miR-221/222, miR-24 and miR-191 levels were all decreased in the BM during $P v$ infection, and returned to normal during convalescence (Figure 1c). In contrast, miR-144 and miR150 levels were both upregulated during infection (Figure 1c) (Baro et al., 2017). However, these findings need further validation using in vitro models validated with clinical samples. Indeed, an in vitro model of tridimensional co-culture in a Matrigel scaffold with Pf gametocytes and self-assembling spheroids of human bone marrow mesenchymal cells (hBM-MSCs) was recently described (Messina et al., 2018), where the immature gametocytes adhered to hBM-MSCs via trypsin-sensitive parasite ligands exposed on the erythrocyte surface (Messina et al., 2018). This model could be used to identify altered miRNA expression by comparing the models 
with and without gametocytes, prior to validating these findings in infected individuals.

\section{OTHER RELEVANT CONSIDERATIONS}

\section{Age, Immunity, and miRNAs}

Falciparum malaria-associated mortality is higher in children under five years of age, who lack immunity to the parasite (Streatfield et al., 2014). In contrast, adults in endemic areas have been exposed regularly, and have progressively built up an anti-disease immunity leading to sub-clinical infections (Schwartz et al., 2001). Because the development of such immunity depends on malaria exposure, SM cases are more often seen in African children as high malaria transmission intensity in sub-Saharan Africa leads to develop antimalarial immunity during childhood. However, in South East Asia, where malaria transmission is not sufficiently intense to induce robust immunity, SM mainly affects older children and adults (Sahu et al., 2015). Therefore, biomarkers of malaria infection validated in both age groups and transmission intensities would represent a powerful prognosis tool to identify patients at risk of SM fatality, inform their clinical management, and decrease SM associated mortality. In the context of miRNA-based biomarkers, miR-3158-3p is the only candidate that has been associated with SM and subcategories of SM in both Mozambican children (Gupta et al., 2021a), as well as Indian adults and children (Gupta et al., 2021b). Further validations and translational efforts are now needed, and the former may also help elucidating the mechanisms behind distinct the different clinical profiles of SM found in African children and South East Asian adults (Wassmer et al., 2015).

\section{Technical Considerations}

Studies investigating the potential diagnostic benefit of miRNAbased biomarkers in SM all used samples obtained patients in malaria endemic countries where co-infections are frequent. Because the symptoms of malaria infection are non-specific, they can easily overlap with concurrent infections, and one important consideration for future studies is to ensure that miRNAs candidates have been tested against non-malarial diseases. Indeed, the observed miRNA level changes in malaria patients can be influenced by inflammation due to the presence of other pathogens and samples obtained from patients with non-malarial diseases should be used as additional controls. There are several techniques available for the identification of miRNAs (recently reviewed in (Sempere et al.; Condrat et al., 2020) including classical and low-throughput methods such as northern blot and RNA protection assays, requiring large quantities of total RNA, and advanced methods such as microarray, nCounter Nanostring technology, NGS and reverse transcription quantitative PCR (RT-qPCR). Among these, microarray, nCounter Nanostring technology and NGS are usually used in the discovery phase or initial screening of the study, while RT-qPCR assays are used in the validation phase (Sempere et al.; Condrat et al., 2020). However, the use of RT-
qPCRs can be limited due to the lack of appropriate normalizing controls. In our opinion, endogenous miRNA controls (ECs) should be disease-specific as ECs with stable expression in the case and control groups found suitable for cancer studies may not be appropriate for malaria samples. A study reported the combination of hsa-miR-30d-5p and hsa-miR-191-5p as the suitable ECs to normalize the miRNA RT-qPCRs data obtained using plasma from patients with malaria infection (Gupta et al., 2021a). The combination of ECs had a 0.044 NormFinder stability value. In addition, no differences were found in $\mathrm{Ct}$ values of the two ECs when compared between Mozambican patients with SM and UM (Gupta et al., 2021a), further confirming their stable expression in different malaria pathologies.

\section{Future Directions}

Lateral flow assays (LFA) developed to detect circulating miRNAs associated with different type of cancers are showing promising results. A gold nanoparticle-based LFA was able to detect a minimum concentration of $60 \mathrm{pM}$ of miR-215 within 20 minutes in aqueous solutions and biological samples (Gao et al., 2014). Similarly, another LFA was able to detect miR-21, miR155 and miR-210 with detection limits of $0.073,0.061$ and 0.085 nM, respectively (Zheng et al., 2018). Lastly a Gold@Silica nanocomposite-labeled LFA allowed the visual detection of miR-21 in cancer cells and human serum down to $1 \mathrm{pM}$ (Dong et al., 2021). While these concentration thresholds are highly likely to afford the detection of candidates such as miR3158-3p in SM, additional studies using plasma from different groups of infected individuals are needed to assess the sensitivity and reliability of such LFAs in malaria diagnosis. They could represent a game changer in the field, especially when the number of $p$ fhrp2 deletion reports are increasing (Gupta et al., 2017b; Gendrot et al., 2019; Galatas et al., 2020). PfHRP2-based rapid diagnostic tests (RDTs) are the most widely used diagnostic method across malaria endemic countries (Gendrot et al., 2019). The advantage of miRNA-based LFA is that they could be designed to detect multiple miRNAs (Zheng et al., 2018). This would not only further enhance the sensitivity and specificity of the assay, but also allow a higher granularity in clinical diagnosis.

In addition to their potential as diagnostic tools, miRNA may also open new therapeutic avenues in SM, by either mimicking or inhibiting specific miRNAs associated with its pathogenesis. In the former case, miRNA mimics aim to restore the expression of miRNA that was lost. Inversely, anti-miRNAs (antimiRs) are single stranded oligonucleotides, which are chemically designed to block the function of miRNA candidate overexpressed during the course of the disease (Rupaimoole and Slack, 2017). Attempts have been made using miRNA mimics and antimiRs for the therapeutic intervention in other diseases, including a mimic of the tumour suppressor miRNA miR-34, which reached phase I clinical trials for treating cancer, and antimiRs targeted at miR122, which reached phase II trials for treating hepatitis (Rupaimoole and Slack, 2017; Hanna et al., 2019). Similarly, miR-16, miR-21, miR-29, miR-92 and miR-155 based therapeutics are also in phase I and II trials to test their efficacy to cure wound healing, heart failure, cancer and other 
diseases (Hanna et al., 2019). While these approaches are not currently explored in malaria research, further investigations in the role of miR-3158-3p in regulating the genes associated with brain injury and processes relevant to SM may be promising. In addition, the WHO recently endorsed the RTS,S/AS01 (Mosquitix $^{\mathrm{TM}}$ ) malaria vaccine for use among children in subSaharan Africa and in other regions with moderate to high falciparum malaria transmission. Several groups reported that vaccines could influence serum miRNA levels: a study demonstrated a change in miRNA expression levels in samples obtained from UK children who received vaccination against influenza (H1N1) (Drury et al., 2019). Using mice models, another study showed that vaccines associated with or without protection against respiratory syncytial virus led to different circulating miRNA profiles (Atherton et al., 2019). The roll-out of the Mosquitix ${ }^{\mathrm{TM}}$ vaccine will represent a great opportunity to investigate over-expressed or down-regulated miRNAs in protected vaccinated individuals. In turn, these could inform miRNA-based therapeutics against $P f$ infection.

\section{CONCLUSIONS}

Microscopy remains the gold standard for malaria diagnosis. However, no currently available methods can identify patients with parasite sequestration-associated tissue injury or predict their level of infection severity. There is a need for new tools and technologies easily implementable in malarious areas to improve diagnosis and increase survival. miRNAs, which are rapidly

\section{REFERENCES}

Aguilar R., Magallon-Tejada A., Achtman A. H., Moraleda C., Joice R., Cistero P., et al. (2014). Molecular Evidence for the Localization of Plasmodium Falciparum Immature Gametocytes in Bone Marrow. Blood 123 (7), 959966. doi: 10.1182/blood-2013-08-520767

Al-Quraishy S., Dkhil M. A., Delic D., Abdel-Baki A. A., and Wunderlich F. (2012). Organ-Specific Testosterone-Insensitive Response of miRNA Expression of C57BL/6 Mice to Plasmodium Chabaudi Malaria. Parasitol. Res. 111 (3), 1093-1101. doi: 10.1007/s00436-012-2937-3

Andolina C., Rek J. C., Briggs J., Okoth J., Musiime A., Ramjith J., et al. (2021). Sources of Persistent Malaria Transmission in a Setting With Effective Malaria Control in Eastern Uganda: A Longitudinal, Observational Cohort Study. Lancet Infect. Dis. 21 (11), 1568-1578. doi: 10.1016/S1473-3099(21)00072-4

Anvikar A. R., van Eijk A. M., Shah A., Upadhyay K. J., Sullivan S. A., Patel A. J., et al. (2020). Clinical and Epidemiological Characterization of Severe Plasmodium Vivax Malaria in Gujarat, India. Virulence 11 (1), 730-738. doi: 10.1080/21505594.2020.1773107

Arroyo J. D., Chevillet J. R., Kroh E. M., Ruf I. K., Pritchard C. C., Gibson D. F., et al. (2011). Argonaute2 Complexes Carry a Population of Circulating Micrornas Independent of Vesicles in Human Plasma. Proc. Natl. Acad. Sci. U. S. A. 108 (12), 5003-5008. doi: 10.1073/pnas.1019055108

Atherton L. J., Jorquera P. A., Bakre A. A., and Tripp R. A. (2019). Determining Immune and MiRNA Biomarkers Related to Respiratory Syncytial Virus (RSV) Vaccine Types. Front. Immunol. 10, 2323. doi: 10.3389/fimmu.2019.02323

Babatunde K. A., Yesodha Subramanian B., Ahouidi A. D., Martinez Murillo P., Walch M., and Mantel P. Y. (2020). Role of Extracellular Vesicles in Cellular Cross Talk in Malaria. Front. Immunol. 11, 22. doi: 10.3389/fimmu.2020.00022

Baird J. K., Valecha N., Duparc S., White N. J., and Price R. N. (2016). Diagnosis and Treatment of Plasmodium Vivax Malaria. Am. J. Trop. Med. Hyg. 95 (6 Suppl), 35-51. doi: 10.4269/ajtmh.16-0171 released in biofluids upon infection and organ damage, could serve as a measure of tissue injury and be used to accurately detect parasites not only in infected humans but also in mosquitoes, to contribute to malaria elimination efforts.

\section{AUTHOR CONTRIBUTIONS}

HG and SW designed and conceptualized the manuscript. HG carried out the literature search, and together with SW generated the first draft of the manuscript. Both authors reviewed and approved the final manuscript.

\section{FUNDING}

This work was supported by the National Institute of Allergy and Infectious Diseases of the National Institutes of Health under Award Numbers U19AI089676 and R21AI142472, and by the Medical Research Council, UK, under Award Number MR/ S009450/1. The content is solely the responsibility of the authors and does not necessarily represent the official views of the funders.

\section{ACKNOWLEDGMENTS}

We would like to thank Benjamin Wassmer for drawing some of the beautiful artwork in Figure 1.

Barker K. R., Lu Z., Kim H., Zheng Y., Chen J., Conroy A. L., et al. (2017). MiR-155 Modifies Inflammation, Endothelial Activation and Blood-Brain Barrier Dysfunction in Cerebral Malaria. Mol. Med. 23, 24-33. doi: 10.2119/ molmed.2016.00139

Baro B., Deroost K., Raiol T., Brito M., Almeida A. C., De Menezes-Neto A., et al. (2017). Plasmodium Vivax Gametocytes in the Bone Marrow of an Acute Malaria Patient and Changes in the Erythroid Mirna Profile. PloS Negl. Trop. Dis. 11 (4), e0005365. doi: 10.1371/journal.pntd.0005365

Bertrams W., Griss K., Han M., Seidel K., Hippenstiel S., Suttorp N., et al. (2021). Transcriptional Analysis Identifies Potential Biomarkers and Molecular Regulators in Acute Malaria Infection. Life Sci. 270, 119158. doi: 10.1016/ j.lfs.2021.119158

Biryukova I., Ye T., and Levashina E. (2014). Transcriptome-Wide Analysis of Microrna Expression in the Malaria Mosquito Anopheles Gambiae. BMC Genomics 15, 557. doi: 10.1186/1471-2164-15-557

Bousema T., and Drakeley C. (2011). Epidemiology and Infectivity of Plasmodium Falciparum and Plasmodium Vivax Gametocytes in Relation to Malaria Control and Elimination. Clin. Microbiol. Rev. 24 (2), 377-410. doi: 10.1128/ CMR.00051-10

Bousema T., Okell L., Felger I., and Drakeley C. (2014). Asymptomatic Malaria Infections: Detectability, Transmissibility and Public Health Relevance. Nat. Rev. Microbiol. 12 (12), 833-840. doi: 10.1038/nrmicro3364

Brabin B. J., Romagosa C., Abdelgalil S., Menendez C., Verhoeff F. H., Mcgready R., et al. (2004). The Sick Placenta-The Role of Malaria. Placenta 25 (5), 359378. doi: 10.1016/j.placenta.2003.10.019

Buffet P. A., Safeukui I., Deplaine G., Brousse V., Prendki V., Thellier M., et al. (2011). The Pathogenesis of Plasmodium Falciparum Malaria in Humans: Insights From Splenic Physiology. Blood 117 (2), 381-392. doi: 10.1182/blood-2010-04-202911

Bullerdiek J., and Flor I. (2012). Exosome-Delivered Micrornas of "Chromosome 19 MicroRNA Cluster" as Immunomodulators in Pregnancy and Tumorigenesis. Mol. Cytogenet. 5 (1), 27. doi: 10.1186/1755-8166-5-27 
Burel J. G., Apte S. H., Groves P. L., Boyle M. J., Langer C., Beeson J. G., et al. (2017). Dichotomous MiR Expression and Immune Responses Following Primary Blood-Stage Malaria. JCI Insight 2 (15). doi: 10.1172/jci.insight.93434

Calzetta M., Perugini E., Seixas G., Sousa C. A., Guelbeogo W. M., Sagnon N., et al. (2018). A Novel Nested Polymerase Chain Reaction Assay Targeting Plasmodium Mitochondrial DNA in Field-Collected Anopheles Mosquitoes. Med. Vet. Entomol. 32 (3), 372-377. doi: 10.1111/mve.12293

Chamnanchanunt S., Fucharoen S., and Umemura T. (2017). Circulating MicroRNAs in Malaria Infection: Bench to Bedside. Malar. J. 16 (1), 334. doi: 10.1186/s12936-017-1990-x

Chamnanchanunt S., Kuroki C., Desakorn V., Enomoto M., Thanachartwet V., Sahassananda D., et al. (2015). Downregulation of Plasma MiR-451 and MiR16 in Plasmodium Vivax Infection. Exp. Parasitol. 155, 19-25. doi: 10.1016/ j.exppara.2015.04.013

Chen I., Clarke S. E., Gosling R., Hamainza B., Killeen G., Magill A., et al. (2016). "Asymptomatic" Malaria: A Chronic and Debilitating Infection That Should be Treated. PloS Med. 13 (1), e1001942. doi: 10.1371/journal.pmed.1001942

Cheng L., Sharples R. A., Scicluna B. J., and Hill A. F. (2014). Exosomes Provide a Protective and Enriched Source of Mirna for Biomarker Profiling Compared to Intracellular and Cell-Free Blood. J. Extracell. Vesicles 3. doi: 10.3402/ jev.v3.23743

Cheng I. S., Sealy B. C., Tiberti N., and Combes V. (2020) Extracellular Vesicles, From Pathogenesis to Biomarkers: the Case for Cerebral Malaria. Vessel Plus 4, 17. doi: 10.20517/2574-1209.2020.08

Cohen A., Zinger A., Tiberti N., Grau G. E. R., and Combes V. (2018). Differential Plasma Microvesicle and Brain Profiles of MicroRNA in Experimental Cerebral Malaria. Malar. J. 17 (1), 192. doi: 10.1186/s12936-018-2330-5

Combes V., Taylor T. E., Juhan-Vague I., Mege J. L., Mwenechanya J., Tembo M., et al. (2004). Circulating Endothelial Microparticles in Malawian Children With Severe Falciparum Malaria Complicated With Coma. JAMA 291 (21), 2542-2544. doi: 10.1001/jama.291.21.2542-b

Condrat C. E., Thompson D. C., Barbu M. G., Bugnar O. L., Boboc A., Cretoiu D., et al. (2020). Mirnas as Biomarkers in Disease: Latest Findings Regarding Their Role in Diagnosis and Prognosis. Cells 9 (2). doi: 10.3390/cells9020276

Cortez M. A., Bueso-Ramos C., Ferdin J., Lopez-Berestein G., Sood A. K., and Calin G. A. (2011). MicroRNAs in Body Fluids-the Mix of Hormones and Biomarkers. Nat. Rev. Clin. Oncol. 8 (8), 467-477. doi: 10.1038/ nrclinonc. 2011.76

Cox-Singh J., Hiu J., Lucas S. B., Divis P. C., Zulkarnaen M., Chandran P., et al. (2010). Severe Malaria - a Case of Fatal Plasmodium Knowlesi Infection With Post-Mortem Findings: A Case Report. Malar. J. 9, 10. doi: 10.1186/1475-28759-10

Cranston H. A., Boylan C. W., Carroll G. L., Sutera S. P., Williamson J. R., Gluzman I. Y., et al. (1984). Plasmodium Falciparum Maturation Abolishes Physiologic Red Cell Deformability. Science 223 (4634), 400-403. doi: 10.1126/ science.6362007

Dayananda K. K., Achur R. N., and Gowda D. C. (2018). Epidemiology, Drug Resistance, and Pathophysiology of Plasmodium Vivax Malaria. J. Vector Borne Dis. 55 (1), 1-8. doi: 10.4103/0972-9062.234620

Delic D., Dkhil M., Al-Quraishy S., and Wunderlich F. (2011). Hepatic MiRNA Expression Reprogrammed by Plasmodium Chabaudi Malaria. Parasitol. Res. 108 (5), 1111-1121. doi: 10.1007/s00436-010-2152-z

Dennison N. J., BenMarzouk-Hidalgo O. J., and Dimopoulos G. (2015). MicroRNA-Regulation of Anopheles Gambiae Immunity to Plasmodium Falciparum Infection and Midgut Microbiota. Dev. Comp. Immunol. 49 (1), 170-178. doi: 10.1016/j.dci.2014.10.016

Dieng M. M., Diawara A., Manikandan V., Tamim El Jarkass H., Serme S. S., Sombie S., et al. (2020). Integrative Genomic Analysis Reveals Mechanisms of Immune Evasion in P. Falciparum Malaria. Nat. Commun. 11 (1), 5093. doi: 10.1038/s41467-020-18915-6

Dkhil M. A., Al-Quraishy S. A., Abdel-Baki A. S., Delic D., and Wunderlich F. (2016). Differential MiRNA Expression in the Liver of Balb/C Mice Protected by Vaccination During Crisis of Plasmodium Chabaudi Blood-Stage Malaria. Front. Microbiol. 7, 2155. doi: 10.3389/fmicb.2016.02155

Dong S., Fu X., Dong Y., Simoes M. L., Zhu J., and Dimopoulos G. (2020). Broad Spectrum Immunomodulatory Effects of Anopheles Gambiae Micrornas and Their Use for Transgenic Suppression of Plasmodium. PloS Pathog. 16 (4), e1008453. doi: 10.1371/journal.ppat.1008453
Dong T., Yin R., Yu Q., Qiu W., Li K., Qian L., et al. (2021). Sensitive Detection of Microrna-21 in Cancer Cells and Human Serum With Au@Si Nanocomposite and Lateral Flow Assay. Anal. Chim. Acta 1147, 56-63. doi: 10.1016/ j.aca.2020.12.042

Drury R. E., Pollard A. J., and O'Connor D. (2019). The Effect of H1N1 Vaccination on Serum MiRNA Expression in Children: A Tale of Caution for MicroRNA Microarray Studies. PloS One 14 (8), e0221143. doi: 10.1371/ journal.pone.0221143

Echeverry D. F., Deason N. A., Makuru V., Davidson J., Xiao H., Niedbalski J., et al. (2017). Fast and Robust Single PCR for Plasmodium Sporozoite Detection in Mosquitoes Using the Cytochrome Oxidase I Gene. Malar. J. 16 (1), 230. doi: 10.1186/s12936-017-1881-1

EL Andaloussi S., Mager I., Breakefield X. O., and Wood M. J. (2013). Extracellular Vesicles: Biology and Emerging Therapeutic Opportunities. Nat. Rev. Drug Discov. 12 (5), 347-357. doi: 10.1038/nrd3978

El-Assaad F., Hempel C., Combes V., Mitchell A. J., Ball H. J., Kurtzhals J. A., et al. (2011). Differential MicroRNA Expression in Experimental Cerebral and Noncerebral Malaria. Infect. Immun. 79 (6), 2379-2384. doi: 10.1128/ IAI.01136-10

Fleischer B. (2004). Editorial: 100 Years Ago: Giemsa's Solution for Staining of Plasmodia. Trop. Med. Int. Health 9 (7), 755-756. doi: 10.1111/j.13653156.2004.01278.x

Fried M., and Duffy P. E. (1996). Adherence of Plasmodium Falciparum to Chondroitin Sulfate a in the Human Placenta. Science 272 (5267), 1502-1504. doi: 10.1126/science.272.5267.1502

Galatas B., Bassat Q., and Mayor A. (2016). Malaria Parasites in the Asymptomatic: Looking for the Hay in the Haystack. Trends Parasitol. 32 (4), 296-308. doi: 10.1016/j.pt.2015.11.015

Galatas B., Mayor A., Gupta H., Balanza N., Jang I. K., Nhamussua L., et al. (2020). Field Performance of Ultrasensitive and Conventional Malaria Rapid Diagnostic Tests in Southern Mozambique. Malar. J. 19 (1), 451. doi: 10.1186/s12936-020-03526-9

Gamain B., Smith J. D., Viebig N. K., Gysin J., and Scherf A. (2007). PregnancyAssociated Malaria: Parasite Binding, Natural Immunity and Vaccine Development. Int. J. Parasitol. 37 (3-4), 273-283. doi: 10.1016/j.ijpara. 2006.11.011

Gao X., Xu H., Baloda M., Gurung A. S., Xu L. P., Wang T., et al. (2014). Visual Detection of Microrna With Lateral Flow Nucleic Acid Biosensor. Biosens Bioelectron 54, 578-584. doi: 10.1016/j.bios.2013.10.055

Garcia L. S. (2010). Malaria. Clin. Lab. Med. 30 (1), 93-129. doi: 10.1016/ j.cll.2009.10.001

Gazzinelli R. T., Kalantari P., Fitzgerald K. A., and Golenbock D. T. (2014). Innate Sensing of Malaria Parasites. Nat. Rev. Immunol. 14 (11), 744-757. doi: 10.1038/nri3742

Gendrot M., Fawaz R., Dormoi J., Madamet M., and Pradines B. (2019). Genetic Diversity and Deletion of Plasmodium Falciparum Histidine-Rich Protein 2 and 3: A Threat to Diagnosis of P. Falciparum Malaria. Clin. Microbiol. Infect. 25 (5), 580-585. doi: 10.1016/j.cmi.2018.09.009

Gupta H., Afsal M. P., Shetty S. M., Satyamoorthy K., and Umakanth S. (2015a). Plasmodium Vivax Infection Causes Acute Respiratory Distress Syndrome: A Case Report. J. Infect. Dev. Ctries. 9 (8), 910-913. doi: 10.3855/jidc.6813

Gupta H., Chaudhari S., Rai A., Bhat S., Sahu P. K., Hande M. H., et al. (2017a). Genetic and Epigenetic Changes in Host ABCB1 Influences Malaria Susceptibility to Plasmodium Falciparum. PloS One 12 (4), e0175702. doi: 10.1371/journal.pone.0175702

Gupta H., Dhunputh P., Bhatt A. N., Satyamoorthy K., and Umakanth S. (2016a). Cerebral Malaria in a Man With Plasmodium Vivax Mono-Infection: A Case Report. Trop. Doct. 46 (4), 241-245. doi: 10.1177/0049475515624857

Gupta H., Jain A., Saadi A. V., Vasudevan T. G., Hande M. H., D'souza S. C., et al. (2015b). Categorical Complexities of Plasmodium Falciparum Malaria in Individuals Is Associated With Genetic Variations in ADORA2A and GRK5 Genes. Infect. Genet. Evol. 34, 188-199. doi: 10.1016/j.meegid.2015.06.010

Gupta H., Matambisso G., Galatas B., Cistero P., Nhamussua L., Simone W., et al. (2017b). Molecular Surveillance of Pfhrp2 and Pfhrp3 Deletions in Plasmodium Falciparum Isolates From Mozambique. Malar. J. 16 (1), 416. doi: 10.1186/s12936-017-2061-Z

Gupta H., Rubio M., Sitoe A., Cistero P., Nhamussua L., Simone W., et al. (2021a). Plasma MicroRNA Profiling of Plasmodium Falciparum Biomass and 
Association With Severity of Malaria Disease. Emerg. Infect. Dis. 27 (2), 430442. doi: 10.3201/eid2702.191795

Gupta H., Sahu P. K., Pattnaik R., Mohanty A., Majhi M., Mohanty A. K., et al. (2021b). Plasma Levels of Hsa-MiR-3158-3p MicroRNA on Admission Correlate With MRI Findings and Predict Outcome in Cerebral Malaria. Clin. Transl. Med. 11 (6), e396. doi: 10.1002/ctm2.396

Gupta H., Sakharwade S. C., Angural A., Kotambail A., Bhat G. K., Hande M. H., et al. (2013). Evidence for Genetic Linkage Between a Polymorphism in the GNAS Gene and Malaria in South Indian Population. Acta Trop. 128 (3), 571577. doi: 10.1016/j.actatropica.2013.08.005

Gupta H., Srivastava S., Chaudhari S., Vasudevan T. G., Hande M. H., D'souza S. C., et al. (2016b). New Molecular Detection Methods of Malaria Parasites With Multiple Genes From Genomes. Acta Trop. 160, 15-22. doi: 10.1016/ j.actatropica.2016.04.013

Habluetzel A., Merzagora L., Jenni L., Betschart B., Rotigliano G., and Esposito F. (1992). Detecting Malaria Sporozoites in Live, Field-Collected Mosquitoes. Trans. R Soc. Trop. Med. Hyg. 86 (2), 138-140. doi: 10.1016/0035-9203(92) 90542-K

Hanna J., Hossain G. S., and Kocerha J. (2019). The Potential for Microrna Therapeutics and Clinical Research. Front. Genet. 10, 478. doi: 10.3389/ fgene.2019.00478

Hentzschel F., Hammerschmidt-Kamper C., Borner K., Heiss K., Knapp B., Sattler J. M., et al. (2014). AAV8-Mediated In Vivo Overexpression of MiR-155 Enhances the Protective Capacity of Genetically Attenuated Malarial Parasites. Mol. Ther. 22 (12), 2130-2141. doi: 10.1038/mt.2014.172

Hviid L., and Jensen A. T. (2015). Pfemp1 - a Parasite Protein Family of Key Importance in Plasmodium Falciparum Malaria Immunity and Pathogenesis. Adv. Parasitol. 88, 51-84. doi: 10.1016/bs.apar.2015.02.004

Jain S., Rana V., Shrinet J., Sharma A., Tridibes A., Sunil S., et al. (2014). Blood Feeding and Plasmodium Infection Alters the Mirnome of Anopheles Stephensi. PloS One 9 (5), e98402. doi: 10.1371/journal.pone.0098402

Jensen A. R., Adams Y., and Hviid L. (2020). Cerebral Plasmodium Falciparum Malaria: The Role of Pfemp1 in Its Pathogenesis and Immunity, and Pfemp1Based Vaccines to Prevent it. Immunol. Rev. 293 (1), 230-252. doi: 10.1111/ imr. 12807

Kaur H., Sehgal R., Kumar A., Sehgal A., Bansal D., and Sultan A. A. (2018). Screening and Identification of Potential Novel Biomarker for Diagnosis of Complicated Plasmodium Vivax Malaria. J. Transl. Med. 16 (1), 272. doi: 10.1186/s12967-018-1646-9

Ketprasit N., Cheng I. S., Deutsch F., Tran N., Imwong M., Combes V., et al. (2020). The Characterization of Extracellular Vesicles-Derived MicroRNAs in Thai Malaria Patients. Malar. J. 19 (1), 285. doi: 10.1186/s12936-020-03360-z

Kho S., Qotrunnada L., Leonardo L., Andries B., Wardani P. a. I., Fricot A., et al. (2021a). Evaluation of Splenic Accumulation and Colocalization of Immature Reticulocytes and Plasmodium Vivax in Asymptomatic Malaria: A Prospective Human Splenectomy Study. PloS Med. 18 (5), e1003632. doi: 10.1371/ journal.pmed.1003632

Kho S., Qotrunnada L., Leonardo L., Andries B., Wardani P. a. I., Fricot A., et al. (2021b). Hidden Biomass of Intact Malaria Parasites in the Human Spleen. N. Engl. J. Med. 384 (21), 2067-2069. doi: 10.1056/NEJMc2023884

Kimenyi K. M., Wamae K., and Ochola-Oyier L. I. (2019). Understanding P. Falciparum Asymptomatic Infections: A Proposition for a Transcriptomic Approach. Front. Immunol. 10, 2398. doi: 10.3389/fimmu.2019.02398

Kotepui M., Kotepui K. U., Milanez G. D., and Masangkay F. R. (2020a). Severity and Mortality of Severe Plasmodium Ovale Infection: A Systematic Review and Meta-Analysis. PloS One 15 (6), e0235014. doi: 10.1371/journal.pone.0235014

Kotepui M., Kotepui K. U., Milanez G. D., and Masangkay F. R. (2020b). Global Prevalence and Mortality of Severe Plasmodium Malariae Infection: A Systematic Review and Meta-Analysis. Malar. J. 19 (1), 274. doi: 10.1186/ s12936-020-03344-z

Lacerda M. V., Mourao M. P., Coelho H. C., and Santos J. B. (2011). Thrombocytopenia in Malaria: Who Cares? Mem. Inst. Oswaldo Cruz. 106 Suppl 1, 52-63. doi: 10.1590/s0074-02762011000900007

LaMonte G., Philip N., Reardon J., Lacsina J. R., Majoros W., Chapman L., et al. (2012). Translocation of Sickle Cell Erythrocyte MicroRNAs Into Plasmodium Falciparum Inhibits Parasite Translation and Contributes to Malaria Resistance. Cell Host Microbe 12 (2), 187-199. doi: 10.1016/j.chom.2012.06.007
Lampe L., Jentzsch M., Kierszniowska S., and Levashina E. A. (2019). Metabolic Balancing by MiR-276 Shapes the Mosquito Reproductive Cycle and Plasmodium Falciparum Development. Nat. Commun. 10 (1), 5634. doi: 10.1038/s41467-019-13627-y

Latourette M. T., Siebert J. E., Barto R. J. Jr., Marable K. L., Muyepa A., Hammond C. A., et al. (2011). Magnetic Resonance Imaging Research in Sub-Saharan Africa: Challenges and Satellite-Based Networking Implementation. J. Digit. Imaging 24 (4), 729-738. doi: 10.1007/s10278-010-9323-4

Liu W., Hao Z., Huang L., Chen L., Wei Q., Cai L., et al. (2017). Comparative Expression Profile of Micrornas in Anopheles Anthropophagus Midgut After Blood-Feeding and Plasmodium Infection. Parasit. Vectors 10 (1), 86. doi: 10.1186/s13071-017-2027-6

Lucchi N. W., Jain V., Wilson N. O., Singh N., Udhayakumar V., and Stiles J. K. (2011). Potential Serological Biomarkers of Cerebral Malaria. Dis. Markers 31 (6), 327-335. doi: 10.1155/2011/345706

Makani J., Matuja W., Liyombo E., Snow R. W., Marsh K., and Warrell D. A. (2003). Admission Diagnosis of Cerebral Malaria in Adults in an Endemic Area of Tanzania: Implications and Clinical Description. QJM 96 (5), 355-362. doi: $10.1093 /$ qjmed/hcg059

Mantel P. Y., Hjelmqvist D., Walch M., Kharoubi-Hess S., Nilsson S., Ravel D., et al. (2016). Infected Erythrocyte-Derived Extracellular Vesicles Alter Vascular Function via Regulatory Ago2-MiRNA Complexes in Malaria. Nat. Commun. 7, 12727. doi: 10.1038/ncomms 12727

Mantel P. Y., and Marti M. (2014). The Role of Extracellular Vesicles in Plasmodium and Other Protozoan Parasites. Cell Microbiol. 16 (3), 344-354. doi: $10.1111 / \mathrm{cmi} .12259$

Martin-Alonso A., Cohen A., Quispe-Ricalde M. A., Foronda P., Benito A., Berzosa P., et al. (2018). Differentially Expressed MicroRNAs in Experimental Cerebral Malaria and Their Involvement in Endocytosis, Adherens Junctions, Foxo and TGF-Beta Signalling Pathways. Sci. Rep. 8 (1), 11277. doi: 10.1038/s41598-018-29721-y

Maude R. J., Barkhof F., Hassan M. U., Ghose A., Hossain A., Abul Faiz M., et al. (2014). Magnetic Resonance Imaging of the Brain in Adults With Severe Falciparum Malaria. Malar. J. 13, 177. doi: 10.1186/1475-2875-13-177

Mead E. A., and Tu Z. (2008). Cloning, Characterization, and Expression of MicroRNAs From the Asian Malaria Mosquito, Anopheles Stephensi. BMC Genomics 9, 244. doi: 10.1186/1471-2164-9-244

Messina V., Valtieri M., Rubio M., Falchi M., Mancini F., Mayor A., et al. (2018). Gametocytes of the Malaria Parasite Plasmodium Falciparum Interact With and Stimulate Bone Marrow Mesenchymal Cells to Secrete Angiogenetic Factors. Front. Cell. Infect. Microbiol. 8, 50. doi: 10.3389/fcimb.2018.00050

Miller L. H., Baruch D. I., Marsh K., and Doumbo O. K. (2002). The Pathogenic Basis of Malaria. Nature 415 (6872), 673-679. doi: 10.1038/415673a

Milner D. A. Jr., Whitten R. O., Kamiza S., Carr R., Liomba G., Dzamalala C., et al. (2014). The Systemic Pathology of Cerebral Malaria in African Children. Front. Cell. Infect. Microbiol. 4, 104. doi: 10.3389/fcimb.2014.00104

Mischlinger J., Ronnberg C., Alvarez-Martinez M. J., Buhler S., Paul M., Schlagenhauf P., et al. (2020). Imported Malaria in Countries Where Malaria Is Not Endemic: A Comparison of Semi-Immune and Nonimmune Travelers. Clin. Microbiol. Rev. 33. doi: 10.1128/CMR.00104-19

Mohanty S., Mishra S. K., Patnaik R., Dutt A. K., Pradhan S., Das B., et al. (2011). Brain Swelling and Mannitol Therapy in Adult Cerebral Malaria: A Randomized Trial. Clin. Infect. Dis. 53 (4), 349-355. doi: 10.1093/cid/cir405

Mohanty S., Taylor T. E., Kampondeni S., Potchen M. J., Panda P., Majhi M., et al. (2014). Magnetic Resonance Imaging During Life: The Key to Unlock Cerebral Malaria Pathogenesis? Malar. J. 13, 276. doi: 10.1186/1475-2875-13-276

Moody A. H., and Chiodini P. L. (2002). Non-Microscopic Method for Malaria Diagnosis Using Optimal it, a Second-Generation Dipstick for Malaria Pldh Antigen Detection. Br. J. BioMed. Sci. 59 (4), 228-231. doi: 10.1080/ 09674845.2002.11783665

Moro L., Bardaji A., Macete E., Barrios D., Morales-Prieto D. M., Espana C., et al. (2016). Placental Microparticles and Micrornas in Pregnant Women With Plasmodium Falciparum or HIV Infection. PloS One 11 (1), e0146361. doi: 10.1371/journal.pone. 0146361

Mousa A., Al-Taiar A., Anstey N. M., Badaut C., Barber B. E., Bassat Q., et al. (2020). The Impact of Delayed Treatment of Uncomplicated P. Falciparum Malaria on Progression to Severe Malaria: A Systematic Review and a Pooled 
Multicentre Individual-Patient Meta-Analysis. PloS Med. 17 (10), e1003359. doi: 10.1371/journal.pmed.1003359

Nantakomol D., Dondorp A. M., Krudsood S., Udomsangpetch R., Pattanapanyasat K., Combes V., et al. (2011). Circulating Red Cell-Derived Microparticles in Human Malaria. J. Infect. Dis. 203 (5), 700-706. doi: 10.1093/ infdis/jiq104

Nik Mohamed Kamal N., and Shahidan W. N. S. (2019). Non-Exosomal and Exosomal Circulatory MicroRNAs: Which Are More Valid as Biomarkers? Front. Pharmacol. 10, 1500. doi: 10.3389/fphar.2019.01500

Nosten F., Rogerson S. J., Beeson J. G., McGready R., Mutabingwa T. K., and Brabin B. (2004). Malaria in Pregnancy and the Endemicity Spectrum: What can We Learn? Trends Parasitol. 20 (9), 425-432. doi: 10.1016/ j.pt.2004.06.007

Oakley M. S., Gerald N., McCutchan T. F., Aravind L., and Kumar S. (2011). Clinical and Molecular Aspects of Malaria Fever. Trends Parasitol. 27 (10), 442-449. doi: 10.1016/j.pt.2011.06.004

Okell L. C., Bousema T., Griffin J. T., Ouedraogo A. L., Ghani A. C., and Drakeley C. J. (2012). Factors Determining the Occurrence of Submicroscopic Malaria Infections and Their Relevance for Control. Nat. Commun. 3, 1237. doi: $10.1038 /$ ncomms 2241

Ramirez A. L., van den Hurk A. F., Mackay I. M., Yang A. S. P., Hewitson G. R., et al. (2019). Malaria Surveillance From Both Ends: Concurrent Detection of Plasmodium Falciparum in Saliva and Excreta Harvested From Anopheles Mosquitoes. Parasit. Vectors 12 (1), 355. doi: 10.1186/s13071019-3610-9

Rathjen T., Nicol C., McConkey G., and Dalmay T. (2006). Analysis of Short Rnas in the Malaria Parasite and its Red Blood Cell Host. FEBS Lett. 580 (22), 51855188. doi: 10.1016/j.febslet.2006.08.063

Rogerson S. J., Desai M., Mayor A., Sicuri E., Taylor S. M., and van Eijk A. M. (2018). Burden, Pathology, and Costs of Malaria in Pregnancy: New Developments for an Old Problem. Lancet Infect. Dis. 18 (4), e107-e118. doi: 10.1016/S1473-3099(18)30066-5

Rowe J. A., Claessens A., Corrigan R. A., and Arman M. (2009). Adhesion of Plasmodium Falciparum-Infected Erythrocytes to Human Cells: Molecular Mechanisms and Therapeutic Implications. Expert Rev. Mol. Med. 11, e16. doi: $10.1017 /$ S1462399409001082

Rubio M., Bassat Q., Estivill X., and Mayor A. (2016). Tying Malaria and MicroRNAs: From the Biology to Future Diagnostic Perspectives. Malar. J. 15, 167. doi: 10.1186/s12936-016-1222-9

Rupaimoole R., and Slack F. J. (2017). MicroRNA Therapeutics: Towards a New Era for the Management of Cancer and Other Diseases. Nat. Rev. Drug Discov. 16 (3), 203-222. doi: 10.1038/nrd.2016.246

Sahu P. K., Hoffmann A., Majhi M., Pattnaik R., Patterson C., Mahanta K. C., et al. (2020). Brain Magnetic Resonance Imaging Reveals Different Courses of Disease in Pediatric and Adult Cerebral Malaria. Clin. Infect. Dis. 73 (7), e2387-e2396. doi: 10.1093/cid/ciaa1647

Sahu P. K., Satpathi S., Behera P. K., Mishra S. K., Mohanty S., and Wassmer S. C. (2015). Pathogenesis of Cerebral Malaria: New Diagnostic Tools, Biomarkers, and Therapeutic Approaches. Front. Cell Infect. Microbiol. 5, 7.5. doi: 10.3389/ fcimb.2015.00075

Salanti A., Staalsoe T., Lavstsen T., Jensen A. T., Sowa M. P., Arnot D. E., et al. (2003). Selective Upregulation of a Single Distinctly Structured Var Gene in Chondroitin Sulphate a-Adhering Plasmodium Falciparum Involved in Pregnancy-Associated Malaria. Mol. Microbiol. 49 (1), 179-191. doi: 10.1046/j.1365-2958.2003.03570.x

Santos M. L. S., Coimbra R. S., Sousa T. N., Guimaraes L. F. F., Gomes M. S., Amaral L. R., et al. (2021). The Interface Between Inflammatory Mediators and Micrornas in Plasmodium Vivax Severe Thrombocytopenia. Front. Cell. Infect. Microbiol. 11, 631333. doi: 10.3389/fcimb.2021.631333

Schwartz E., Sadetzki S., Murad H., and Raveh D. (2001). Age as a Risk Factor for Severe Plasmodium Falciparum Malaria in Nonimmune Patients. Clin. Infect. Dis. 33 (10), 1774-1777. doi: 10.1086/322522

Sempere L. F., Azmi A. S., and Moore A. (2021). Microrna-Based Diagnostic and Therapeutic Applications in Cancer Medicine. Wiley Interdiscip. Rev. RNA. 2021, e1662. doi: 10.1002/wrna.1662

Seydel K. B., Kampondeni S. D., Valim C., Potchen M. J., Milner D. A., Muwalo F. W., et al. (2015). Brain Swelling and Death in Children With Cerebral Malaria. N. Engl. J. Med. 372 (12), 1126-1137. doi: 10.1056/NEJMoa1400116
Snounou G., Viriyakosol S., Jarra W., Thaithong S., and Brown K. N. (1993). Identification of the Four Human Malaria Parasite Species in Field Samples by the Polymerase Chain Reaction and Detection of a High Prevalence of Mixed Infections. Mol. Biochem. Parasitol. 58 (2), 283-292. doi: 10.1016/0166-6851 (93) $90050-8$

Spencer H. C., Collins W. E., Chin W., and Skinner J. C. (1979). The EnzymeLinked Immunosorbent Assay (ELISA) for Malaria. I. The Use of In VitroCultured Plasmodium Falciparum as Antigen. Am. J. Trop. Med. Hyg. 28 (6), 927-932. doi: 10.4269/ajtmh.1979.28.927

Streatfield P. K., Khan W. A., Bhuiya A., Hanifi S. M., Alam N., Diboulo E., et al. (2014). Malaria Mortality in Africa and Asia: Evidence From INDEPTH Health and Demographic Surveillance System Sites. Glob Health Action 7, 25369. doi: 10.3402/gha.v7.25369

Taylor T. E., Fu W. J., Carr R. A., Whitten R. O., Mueller J. S., Fosiko N. G., et al. (2004). Differentiating the Pathologies of Cerebral Malaria by Postmortem Parasite Counts. Nat. Med. 10 (2), 143-145. doi: 10.1038/ nm986

Tjitra E., Anstey N. M., Sugiarto P., Warikar N., Kenangalem E., Karyana M., et al. (2008). Multidrug-Resistant Plasmodium Vivax Associated With Severe and Fatal Malaria: A Prospective Study in Papua, Indonesia. PloS Med. 5 (6), e128. doi: 10.1371/journal.pmed.0050128

Turner L., Lavstsen T., Berger S. S., Wang C. W., Petersen J. E., Avril M., et al. (2013). Severe Malaria Is Associated With Parasite Binding to Endothelial Protein C Receptor. Nature 498 (7455), 502-505. doi: 10.1038/ nature 12216

Tusting L. S., Bousema T., Smith D. L., and Drakeley C. (2014). Measuring Changes in Plasmodium Falciparum Transmission: Precision, Accuracy and Costs of Metrics. Adv. Parasitol. 84, 151-208. doi: 10.1016/B978-0-12-8000991.00003-X

Valadi H., Ekstrom K., Bossios A., Sjostrand M., Lee J. J., and Lotvall J. O. (2007). Exosome-Mediated Transfer of Mrnas and MicroRNAs Is a Novel Mechanism of Genetic Exchange Between Cells. Nat. Cell Biol. 9 (6), 654-659. doi: 10.1038/ ncb1596

van Loon W., Gai P. P., Hamann L., Bedu-Addo G., and Mockenhaupt F. P. (2019). MiRNA-146a Polymorphism Increases the Odds of Malaria in Pregnancy. Malar. J. 18 (1), 7. doi: 10.1186/s12936-019-2643-Z

Vojtech L., Woo S., Hughes S., Levy C., Ballweber L., Sauteraud R. P., et al. (2014). Exosomes in Human Semen Carry a Distinctive Repertoire of Small nonCoding RNAs With Potential Regulatory Functions. Nucleic Acids Res. 42 (11), 7290-7304. doi: 10.1093/nar/gku347

Wang Z., Xi J., Hao X., Deng W., Liu J., Wei C., et al. (2017). Red Blood Cells Release Microparticles Containing Human Argonaute 2 and miRNAs to Target Genes of Plasmodium Falciparum. Emerg. Microbes Infect. 6 (8), e75. doi: 10.1038/emi.2017.63

Wassmer S. C., Taylor T. E., Rathod P. K., Mishra S. K., Mohanty S., ArevaloHerrera M., et al. (2015). Investigating the Pathogenesis of Severe Malaria: A Multidisciplinary and Cross-Geographical Approach. Am. J. Trop. Med. Hyg. 93 (3 Suppl), 42-56. doi: 10.4269/ajtmh.14-0841

White N. J. (2017). Malaria Parasite Clearance. Malar. J. 16 (1), 88. doi: 10.1186/ s12936-017-1731-1

White N. J., Pukrittayakamee S., Hien T. T., Faiz M. A., Mokuolu O. A., and Dondorp A. M. (2014). Malaria. Lancet 383 (9918), 723-735. doi: 10.1016/ S0140-6736(13)60024-0

White N. J., Turner G. D., Day N. P., and Dondorp A. M. (2013). Lethal Malaria: Marchiafava and Bignami Were Right. J. Infect. Dis. 208 (2), 192-198. doi: 10.1093/infdis/jit116

WHO (2014). Severe Malaria. Trop. Med. Int. Health 19 (Suppl 1), 7-131. doi: 10.1111/tmi.12313_2

WHO (2020). World Malaria Report 2020. Available at: https://www.who.int/ publications/i/item/9789240015791 (Accessed Accessed 09/03/2021).

WHO (2015) Guidelines for the Treatment of Malaria - 3rd Edition (Geneva: World Health Organization). Available at: https://apps.who.int/iris/handle/ 10665/162441 (Accessed Accessed 16/09/2021).

Winter F., Edaye S., Huttenhofer A., and Brunel C. (2007). Anopheles Gambiae Mirnas as Actors of Defence Reaction Against Plasmodium Invasion. Nucleic Acids Res. 35 (20), 6953-6962. doi: 10.1093/nar/gkm686

Wirtz R. A., Burkot T. R., Graves P. M., and Andre R. G. (1987). Field Evaluation of Enzyme-Linked Immunosorbent Assays for Plasmodium Falciparum and 
Plasmodium Vivax Sporozoites in Mosquitoes (Diptera: Culicidae) From Papua New Guinea. J. Med. Entomol. 24 (4), 433-437. doi: 10.1093/jmedent/ 24.4.433

Xue X., Zhang Q., Huang Y., Feng L., and Pan W. (2008). No MiRNA Were Found in Plasmodium and the Ones Identified in Erythrocytes Could Not be Correlated With Infection. Malar. J. 7, 47. doi: 10.1186/14752875-7-47

Zakama A. K., Ozarslan N., and Gaw S. L. (2020). Placental Malaria. Curr. Trop. Med. Rep. 2020, 1-10. doi: 10.1007/s40475-020-00213-2

Zheng W., Yao L., Teng J., Yan C., Qin P., Liu G., et al. (2018). Lateral Flow Test for Visual Detection of Multiple Micrornas. Sens Actuators B Chem. 264, 320326. doi: 10.1016/j.snb.2018.02.159

Zoller T., Naucke T. J., May J., Hoffmeister B., Flick H., Williams C. J., et al. (2009). Malaria Transmission in Non-Endemic Areas: Case Report, Review of the Literature and Implications for Public Health Management. Malar. J. 8, 71. doi: $10.1186 / 1475-2875-8-71$
Conflict of Interest: The authors declare that the research was conducted in the absence of any commercial or financial relationships that could be construed as a potential conflict of interest.

Publisher's Note: All claims expressed in this article are solely those of the authors and do not necessarily represent those of their affiliated organizations, or those of the publisher, the editors and the reviewers. Any product that may be evaluated in this article, or claim that may be made by its manufacturer, is not guaranteed or endorsed by the publisher.

Copyright (C) 2021 Gupta and Wassmer. This is an open-access article distributed under the terms of the Creative Commons Attribution License (CC BY). The use, distribution or reproduction in other forums is permitted, provided the original author(s) and the copyright owner(s) are credited and that the original publication in this journal is cited, in accordance with accepted academic practice. No use, distribution or reproduction is permitted which does not comply with these terms. 